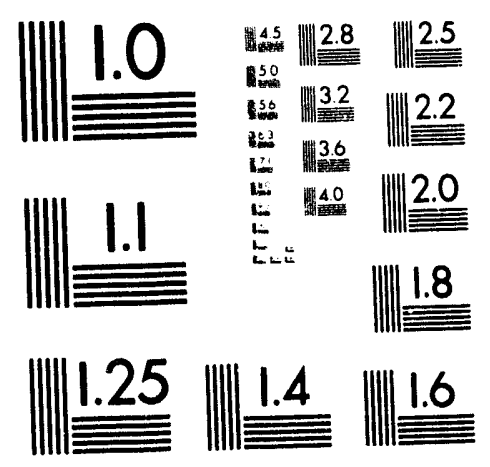



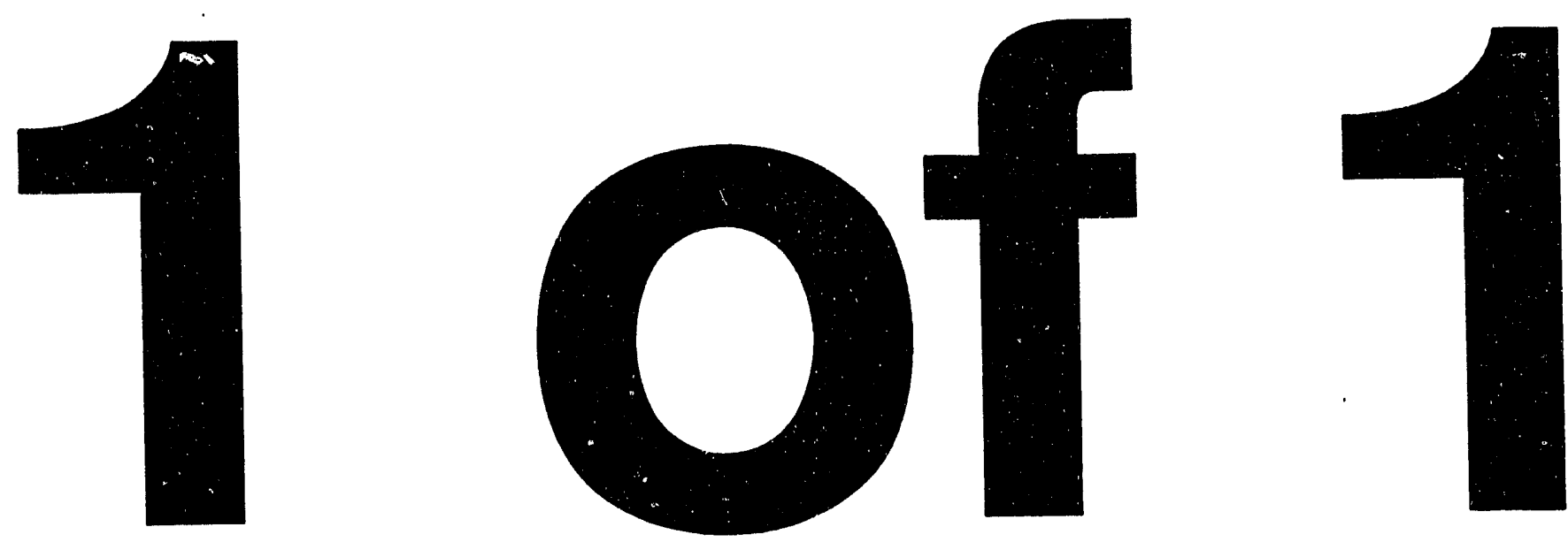
UCRL-JC-114801

PREPRINT

\title{
LOCALLY ADAPTIVE REMESHING SCHEME FOR CALCULATING FLUID INSTABILITIES
}

\author{
G.D. Kramer \\ W.P. Crowley \\ V.C. Rupert \\ This paper was prepared \\ for submittal to the \\ Proceedings of the 19in International Symposium on Shock Waves \\ Marseille, France \\ July 26-30, 1993
}

July 1993

This is a preprint of a paper intended for publication in a journal or proceedings. Since changes may be made before publication, this preprint is made available with the understanding that it will not be cited or reproduced without the permission of the author. 


\section{DISCLAIMER}

This document was prepared as an account of work sponsored by an agency of the United States Government. Neither the United States Government nor the University of Califomia nor any of their employees, makes any warranty, express or implied, or assumes any legal liability or responsibility for the accuracy, completeness, or usefulness of any information, apparatus, product, or process disclosed, or represents that its use would not infringe privately owned rights. Reference herein to any specific commercial products, process, or service by trade name, trademark, manufacturer, or otherwise, does not necessarily constitute or imply its endorsement, recommendation, or favoring by the United States Government or the University of California. The views and opinions of authors expressed herein do not necessarily state or reflect those of the United States Govemment or the University of Califomia, and shall not be used for advertising or product endorsement purposes. 


\section{LOCALLY ADAPTIVE REMESHING SCHEME FOR CALCULATING FLUID INSTABILITIES}

GD Kramer, WP Crowley, and VC Rupert Lawrence Livermore National Laboratory Livermore, CA, USA

\section{Introduction}

LAM is a two dimensional Eulerian hydro code with local adaption that uses a Van Leer limiter, artificial viscosity, and a staggered mesh (Crowley 1992). It differs from the Godonov schemes used elsewhere (Colella and Graz 1985), but this formulation makes it easier to add additional physics to the code. Two distinct advantages of the present code are a conformal quadrilateral mesh option and a grow capability. The code is presently being extended to three dimensions.

\section{Main features of LAM}

Local adaption is a major improvement over past Eulerian codes in that instead of constant zoning throughout the entire problem, zones are added where they are needed and deleted where they are no longer required. The adaption strategy depends on gradients of various quantitics such as density, fractional volume, pressure, energy, or vorticity. For example, the ratio of the density and its first spatial derivative is calculated for each zone. This ratio is then compared with the zonc's size and a user defined accuracy criterion to determine whether resolution or dezoning is required. The level of adaption is defined by the user; we have used 4 as our upper limit. A zone is divided into four equal zones at each level of adaption so the mesh can be dramatically refined in areas where quantitics are changing.

The conformal mesh permits the zoning around an interface to approximate its shape so that there are no zones with partial mass or volume fractions at the start of the calculation. This differs from the usial orthogonal mesh that has mixed zones along the interface between two materials. The two mesh options are shown in Figure 1 for a level 2 adaption.

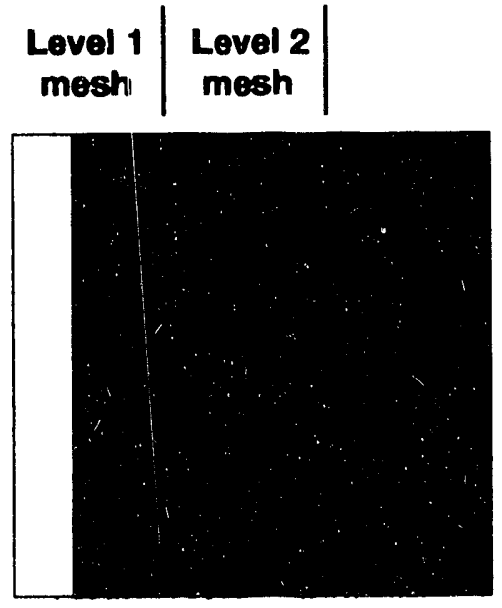

Conformal mesh $\leftarrow$ Shock direction

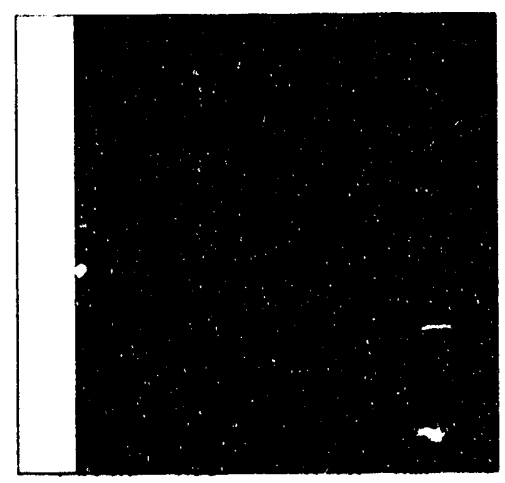

Orthogonal mesh

Fig. 1. LAM with adaption level $=2$. The zoning at left stops at $-3 \mathrm{~cm}$; it can grow from there. 
The grow option is a boundary condition that minimizes the number of zones required for a calculation. Along the grow boundary the mesh remains fixed until the code senses a signal approaching from the interior. The mesh then expands perpendicular to the boundary by adding new unperturbed zones. This process may be repeated many times during a calculation; however, each time the computational domain becomes larger. This can be seen in Figure 1 where the left hand boundary starts at $-3 \mathrm{~cm}$ and grows as the shock approaches it from the right.

Anticipation is crucial for this method to work. This means that the fine mesh has to be in place before the signal gets there. This is especially truc of a flow moving from a heavy fluid into a light fluid where spikes develop ahead of the interface. To minimize the number of zones in the problem, the user is given options for choosing the extent of the anticipation process.

A comparison between LAM and the AMR code (Berger and Colclla 1989) is shown in Figure 2. In this example, which uses Royaumont test problem \#5 (Bcsnard and Haas 1991), a shock with a Mach number of 1.32 propagates into air and crosses an air/SF6 interface, which initially had a sinusoidal shape. The curves for the two mix boundary positions lic cssentially on top of each other.

\section{Initial conditions}

Schemes such is LAM are particularly useful for studying hydro-distorted flows. Bccausc of our interest in the problem of inertial instabilities (Rayleigh-Taylor and RichtmyerMeshkov), we used LAM to study the Richtmyer-Mcshkov cvolution of a shocked interface. The initial conditions are based on a pair of test problems posed for the Royaumont conference (Besnard and Haas 1991). These simulated a shock tube containing a discontinuous interface between helium and argon. One set had the shock incident from the argon side (i.e., heavy material into light) while the other set had the shock incident from the helium side (i.e., light material into heavy). We considered the case where a single shock passes through the interface. If the shock is incident from the helium side, both the transmitted and reflected waves will be shocks. In this instance, the (computational) shock tube must be very long on both sides of the interface to prevent waves reflected from the boundarics from interacting with the perturbed region. If the shock is incident from the argon side, the reflected wave is an expansion wave. For strong enough incident shocks, the

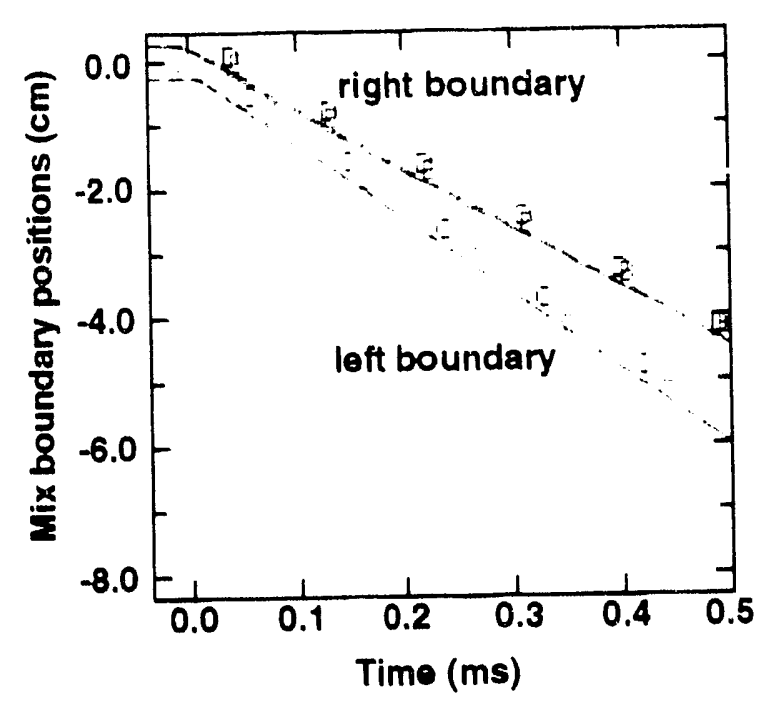

Fig. 2. AMR (a and b) and LAM(d and c) agree for Royauniont test problem \#5. expansion wave will remain on the same side of the original interface position as the transmitted shock. In this case, the shock tube must only be long on the transmittedshock side, resulting in a considerably smaller computational domain if we take advantage of the LAM grow option. For this reason, we have elected to usc the Mach 3.45 shock of the third ICPTM as the nominal initial condition for the $\mathrm{Ar}>\mathrm{He}$ case, with its companion Mach 2.77 shock for the He $>$ Ar case (approximately same interface velocity). The LAM code's grow option allows us to minimize the initial length of the computational domain in the direccion of the transmitted shock. Table 1 lists the initial conditions, and Figure 3 shows the interface shape for some of the 
Table 1. Initial conditions for test problems.

\begin{tabular}{|c|c|c|c|c|}
\hline Pert.\# & 2. Wavenumber & $\operatorname{Min} a / \lambda$ & $\operatorname{Max} a / \lambda$ & Comments \\
\hline 1 & $1-3-4-7-11$ & 0.003 & 0.062 & Royaumont \\
\hline 2 & $1-3-4-7-11$ & 0.006 & 0.074 & Same 5 wavenumbers \\
\hline 3 & $1-3-4-7-11$ & 0.001 & 0.064 & Same 5 wavenumbers \\
\hline 4 & $1-2-3-4-5-6-7-8-9-10-11$ & 0.003 & 0.054 & $\begin{array}{c}\text { More wavenumbers chosen } \\
\text { in the same range }\end{array}$ \\
\hline 5 & $5-7-9-10-11$ & 0.020 & 0.074 & 5 wavenumbers, top of range \\
\hline 6 & 2 & 0.024 & 0.024 & Single wavenumber \\
\hline 7 & $1-2-3-4-5$ & 0.004 & 0.034 & $\begin{array}{c}5 \text { wavenumbers, bottom } \\
\text { range }\end{array}$ \\
\hline 8 & 16 values between $13 \& 89$ & 0.006 & 0.293 & $\begin{array}{l}\text { More wavenumbers, high } \\
\text { range }\end{array}$ \\
\hline
\end{tabular}

problems run. Most of the calculations were run with a combination of wavelengths distributed in the range of those used for the ICPTM. Within this limited range, sets of wave numbers were selected to cover the following cases: single wavelength, small wavelengths, large wavelengths, sample over the range, and the full range of wavelengths. A few calculations were run with a large number of much higher wave numbers to investigate the effect of large amplitude-towavelength ratios, and more intense mode coupling, as can be expected from a real, but nominally flat interface. The latter calculations were more difficult to perform on the conformal mesh, and were run using a rectangular mesh. Results of these calculations are presented in the companion paper by Rupert et al (1993).

\section{Output}

Extracting meaningful information out of runs requires a considerable amount of care. An example of this is the mix width for the $5 \%$ level compared to the width at the $1 \%$ level as shown in Figure 4. Typically mix width is defined as the distance between either the 5 to $95 \%$ or

$$
\text { Ar }>\mathrm{He} M=3.45
$$

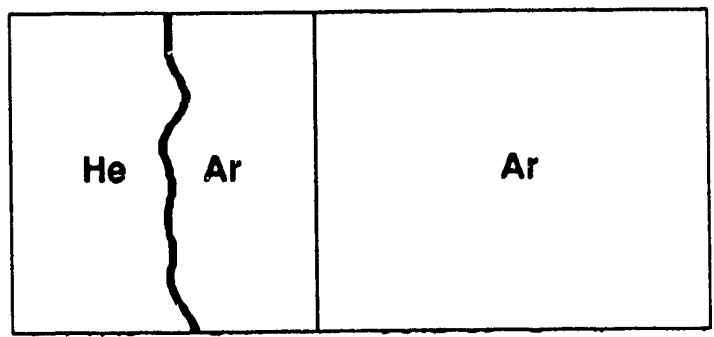

Unshocked
Shocked
He $>$ Ar $M=2.77$

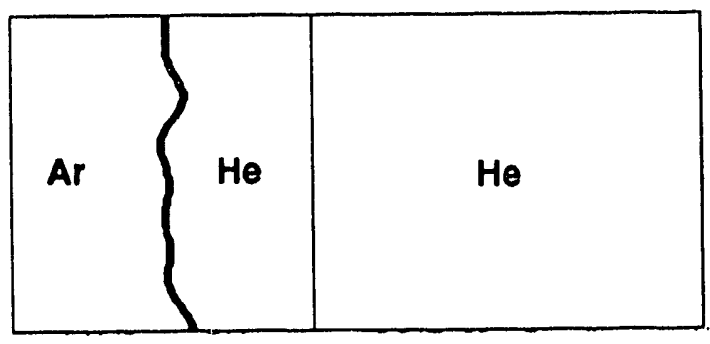

Unshocked
Shocked

Fig. 3. Initial conditions for calculations. The grow boundary moves to the left as the shock approaches it. 


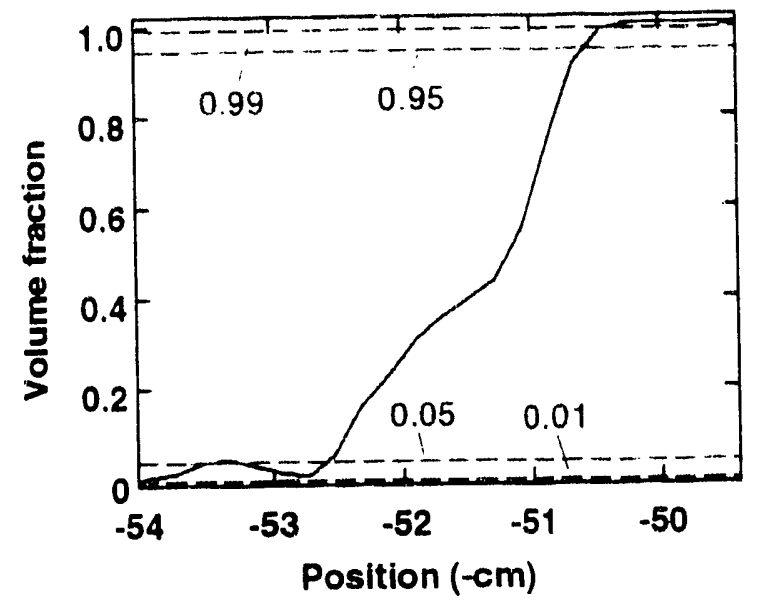

Fig. 4. Mix width for 1 to $99 \%$ and 5 to $95 \%$ with the valley in the volume fraction at $-51.6 \mathrm{~cm}$.

the 1 to $99 \%$ level of the average volume fraction of one material. This average is taken perpendicular to the shock-tube axis. When a spike turns over to form a tree shape (see accompanying paper by Rupert et. al. 1993), valleys occur in the spatial distribution of the average volume fraction, which can cause jumps in the value of the mix width. This can also be seen in Figure 4. Much of the initial work on Richtmyer-Meshkov mixing has concentrated on the mix width, which is a readily obtained experimental quantity. Other diagnostics used are snapshots of density and vulume fraction and the fluctuating part of the kinetic energy calculated in the mix regions. We are investigating means of presenting the data that would lead to additional and hopefully more useful information about the phenomena we seek to simulate, such as the amount of atomic mix, the spectrum of the perturbed region, and the distribution of the fluctuating kinetic energy (FKE) over the whole flow field including the transmitted and reflected waves.

One of the preliminary results we obtained from the expanded diagnostics is that a roughly equal amount of FKE is found at the transmitted shock front, in the reflected expansion wave (heavy-to-light case) and at the interface. A slightly reduced amount is found in the remaining regions. Because of the relative widths of these regions (narrow shock front, wide interface ...) snapshots of the FKE, obtained only around the mix region or in a computational domain not comprising the transmitted and reflected waves, may not give a true picture of the distribution of the fluctuating energy (see accompanying paper of Rupert et. al. 1993).

\section{Summary and future plans}

The LAM code is being used to investigate the interaction of a shock with a perturbed interface between light and heavy materials. We have concentrated on studying the single shock interaction because it sets the stage for further evolution of a multiply shocked interface. The code flexibility has allowed us to decide on the optimum choice of refinement schemes for this particular problem.

All the results presented here were obtained with the two-dimensional version of the code. The three-dimensional version, developed in collaboration between Pat Crowley and Pierric Toulemont is being tested at this time. 
Further additions to the code will encompass high-temperature physics options such as radiation transport, electron transport, non $\gamma$-law EOS, and 3-dimensional modeling, which will allow simulations of additional classes of problems.

\section{References}

Crowley P (1992) A Local Adaptive Mesh Algorithm, Lawrence Livermore National Laboratory, UCRL-JC-109516. Livermore, CA, USA.

Colella P and Graz H M (1985) "Efficient Evolution Algorithms for the Riemann Problem for Real Gases," J. Comp. Phys., vol. 59, no. 2, pp 264-289.

Berger MJ and Colella P (1989) "Local Adaptive Mesh Refinement for Shock Hydrodynamics," J. of Comp. Phys., vol. 82, pp 64-84,

Besnard D and Haas JF (1991) "A Preliminary Review of Numerical Test Problems," Third

International Workshop on the Physics of Compressible Turbulent Mixing, pp 569-590.

Rupert VC, Crowlcy WP, and Kramer GD (1993) "Mix Indiced by Single Shock Passage Through a Material Interface," Proceedings of the $19^{\text {th }}$ International Symposium on Shock Waves, Marseille, France.

Work performed under the auspices of the U.S. Department of Energy by the Lawrence Livermore National Laboratory under Contract W-7405-Eng-48. 

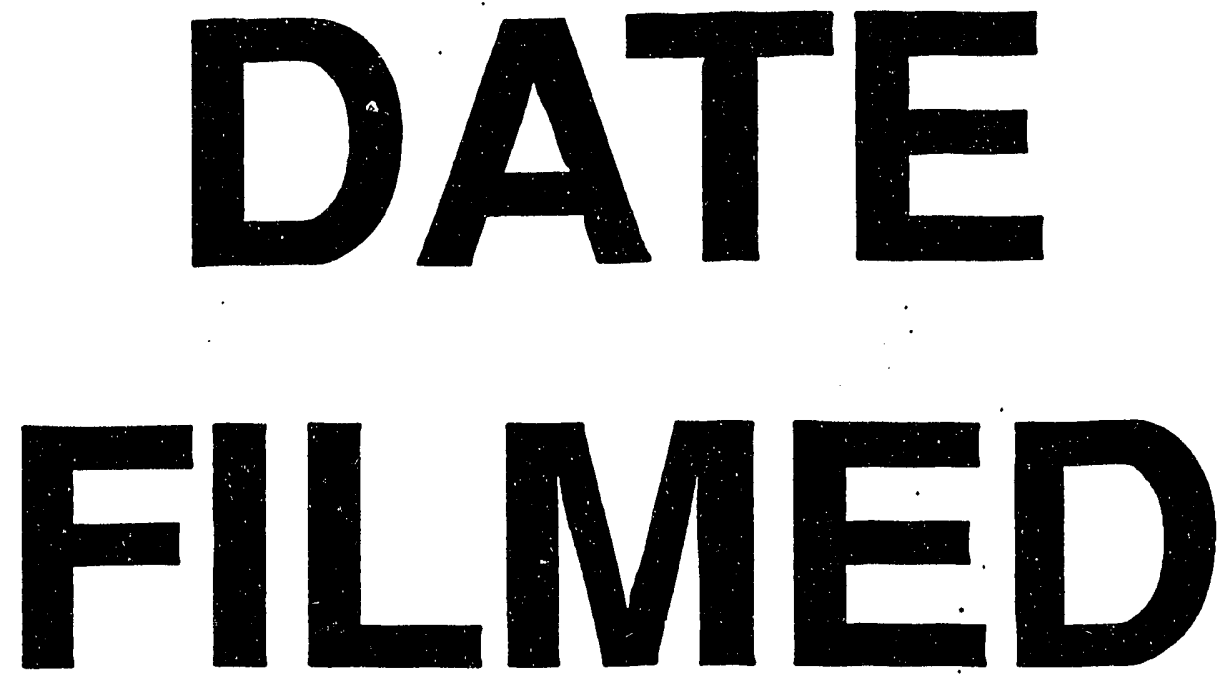

$10 / 19 / 93$
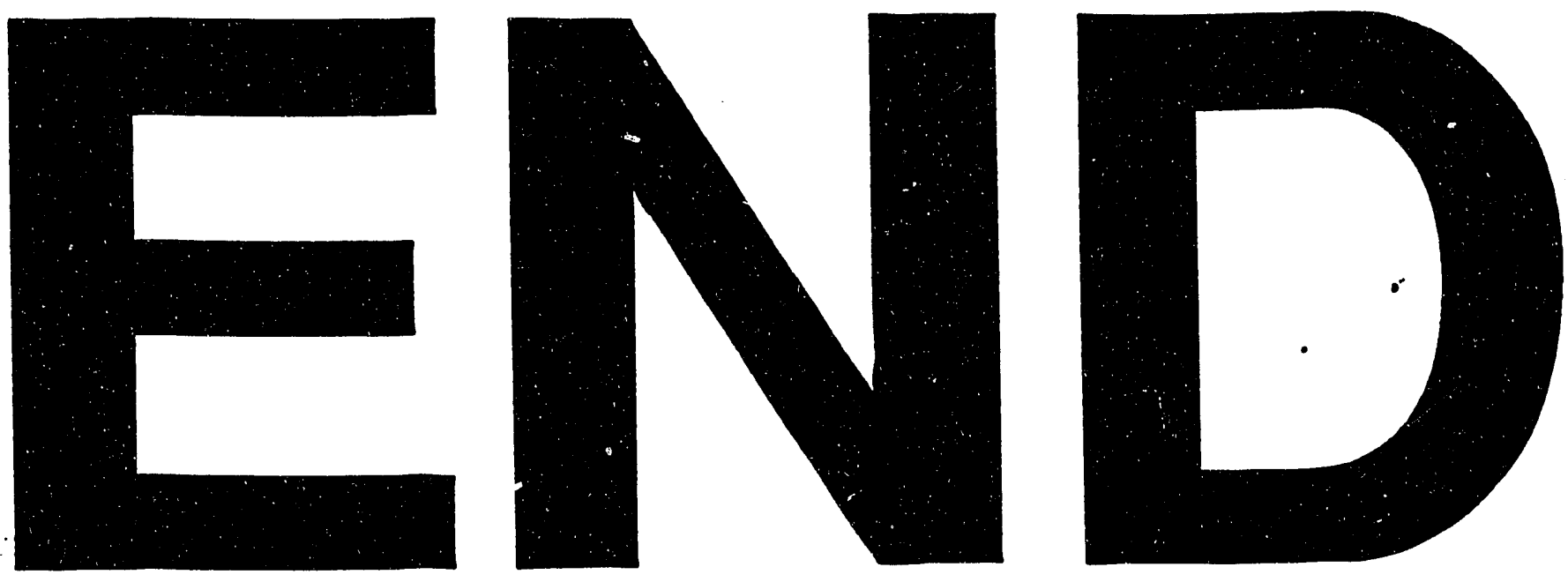
